\title{
How do harpacticoid copepods colonize detrital seagrass leaves?
}

\author{
Thibaud Mascart · Laura Agusto · Gilles Lepoint • \\ François Remy $\cdot$ Marleen De Troch
}

Received: 8 December 2014 / Accepted: 10 February 2015 / Published online: 4 March 2015

(C) Springer-Verlag Berlin Heidelberg 2015

\begin{abstract}
An experiment was carried out investigating the colonization ability and specific pattern of copepods towards a provisional benthic habitat. Since copepods are known to disperse passively and actively, the experiment aimed to investigate the pool of colonizers of macrophytodetritus and the species-specific active colonization pathways. The experiment was performed in a Mediterranean seagrass Posidonia oceanica meadow on defaunated macrophytodetritus accumulations (mainly dead seagrass leaves) for two time intervals ( 24 and $96 \mathrm{~h}$ ). Active colonization by copepods, independently of their adjacent potential source pool habitat (bare sandy sediments, P. oceanica canopy, water column and macrophytodetritus) occurred within $24 \mathrm{~h}$. Natural densities (as in the control treatments) were only reached by active colonization through the water column. Both neither diversities nor species composition of natural macrophytodetritus were ever reached by one single migratory pathway, therefore only a combination of interstitial migration and water column migration can explain the species occurrence under natural condition. Moreover, every potential adjacent source pool habitat contributed species to the newly colonized macrophytodetritus. However, the main colonizers were mostly species with good swimming capabilities. The diverse pool of species present in the newly colonized macrophytodetritus underlines the
\end{abstract}

Communicated by M. Huettel.

T. Mascart $\cdot$ L. Agusto $\cdot$ M. De Troch

Marine Biology, Ghent University, Krijgslaan 281-S8,

9000 Ghent, Belgium

T. Mascart $(\varangle) \cdot$ G. Lepoint $\cdot$ F. Remy

Laboratory of Oceanology, MARE Centre, University of Liège,

Sart Tilman B6C, 4000 Liège, Belgium

e-mail: thibaud.mascart@ugent.be complex communities and dispersion capabilities of copepods. Hence, macrophytodetritus possesses the potential ability to be a colonizer source pool for every adjacent habitat and thus behaves as a copepod hub for the entire seagrass ecosystem.

\section{Introduction}

Dispersion and colonization of new habitats by meiofauna (i.e. the benthic fauna belonging to size class $38 \mu \mathrm{m}-1 \mathrm{~mm}$ ) are highly variable in space and time. This variability is caused by complex interactions between habitat structures, species-specific biological traits, hydrodynamics, resource availability, predation pressure and environmental deterioration (Armonies 1994; Commito and Tita 2002; Bostrom et al. 2010). Since meiofauna lack planktonic larvae (Hagerman and Rieger 1981; Huys and Boxshall 1991), the dispersion mode of adults might be crucial for population dispersion. On small scales (up to a metre), early studies revealed horizontal migration through the sediments interstices as primary mean for meiofauna colonization (i.e. infaunal migration) (McIntyre 1969). However, most of the meiofauna lives on the sediment-water interface being capable of active swimming and infaunal migration on short distances during low water flow (Fleeger et al. 1995). These are thus susceptible to passive erosion and are therefore classified as passive dispersers on larger scales (Palmer 1988; Armonies 1994; Sun and Fleeger 1994; Fleeger et al. 1995). Similar trends for passive dispersal are seen in lotic freshwater ecosystems (Palmer and Gust 1985) and soft-bottom coastal ecosystems with regular tidal currents and strong hydrodynamic forces (Sedlacek and Thistle 2006). Nevertheless, in hydrodynamic calm environments (low flow or with biogenic structures reducing the flow), 
meiobenthic organisms were found suspended in the nearbottom waters revealing active emergence, especially on a diurnal cycle at the onset of dusk (Fleeger et al. 1984; Hicks 1986; Walters 1991; Teasdale et al. 2004). Morphological characteristics were put forward to endorse the emergence availability of phytal and epibenthic meiofauna (Bell et al. 1987; Thistle and Sedlacek 2004; Sedlacek and Thistle 2006). Lower taxonomic classification seems thus to be irrelevant in predicting the habitat utilization of copepods (Sedlacek and Thistle 2006). Noodt (1971) attempted a provisional classification of Copepoda based on the variety of evolved ecological forms (Remane 1952). He highlighted different trends in specialization of eco-morphological characteristics, distinguishing various types of copepod adapted to certain conditions of various habitats (e.g. sediment-living, phytal-living, pelagic). Subsequently, a preferred habitat could be deduced from eco-morphological characteristics. However, a classification of specific copepod colonization abilities is still missing, conversely to nematodes (Bongers 1990). Nowadays, phytal and epibenthic copepods are mainly classified as active dispersers and sedimentary copepods as passive dispersers (Hicks 1986; Kurdziel and Bell 1992).

Though, in case of colonization of new habitat or in the habitat connectivity context, the exact habitat source pool of the colonizers is often unknown. Several studies documented copepods' colonization ability within relatively stable habitats, such as coral fragments (Gheerardyn et al. 2009; Callens et al. 2012), hard substrates (Thomsen et al. 2011), coastal soft sediments (Thielemans and Heip 1984; Scheef and Marcus 2010) and deep-sea sediments (Thistle 1978; Guidi-Guilvard et al. 2009). However, few studies tackled the colonization of provisional habitats such as temporary ponds (Frisch and Green 2007), marine snow aggregates (Kiorboe 2000; Koski et al. 2005) or floating vegetal material (Faust and Gulledge 1996; Ólafsson et al. 2001). The present in situ experiment investigated the colonization of provisional dead seagrass detritus, hereafter referred to as macrophytodetritus. The majority of the macrophytodetritus accumulates on bare sand patches close to seagrass meadow and is decomposed within a few days to several months, depending on the chemical composition and biotic and abiotic fragmentation speed (Romero et al. 1992; Mateo and Romero 1997). These accumulations thereby support high values of secondary production in the receiving communities (Vetter 1995; Mateo and Romero 1997). The structurally complex macrophytodetritus accumulations seem to facilitate the development of meiofaunal communities in coastal marshes (Sanmarti and Menendez 2007), mangrove forests (Torres-Pratts and Schizas 2007) and seagrass beds (Hicks 1980; Coull and Wells 1983; Mascart et al. 2013). In terms of copepod community, connectivity between these accumulation and other adjacent habitats (i.e. seagrass meadow, water column and sediment) is still unstudied, as well as the colonizing process and specific pattern of this colonization.

To try to tackle these three questions, a field experiment was deployed in order to understand the mode of copepod's colonization of Neptune grass Posidonia oceanica macrophytodetritus. The objectives here were threefold: (1) to assess the species composition, densities and diversity of the colonist's source pool (i.e. the sediment, the water column, the $P$. oceanica canopy or other macrophytodetritus patches); (2) to investigate the rate of active colonization of defaunated seagrass macrophytodetritus from adjacent habitats; and (3) to contribute to our knowledge speciesspecific colonization characteristics.

\section{Materials and methods}

Experimental design and sampling site

The experimental site was located in the Gulf of Calvi, Corsica, northwest Mediterranean $\left(42^{\circ} 35^{\prime} \mathrm{N}, 8^{\circ} 43^{\prime} \mathrm{E}\right)$ near the oceanographic station of STARESO (Station de Recherches Sous-marines et Océanographiques, University of Liège). The site consisted of a sand patch (about $100-200 \mathrm{~m}^{2}$ ) at a depth of $12 \mathrm{~m}$, inside a P.oceanica seagrass meadows, partly covered with macrophytodetritus accumulation (MPD). Macrophytodetritus accumulation (10-20 cm thick) was mostly composed of dead P. oceanica leaves and living uprooted seagrass shoots, typical for the Bay area and the time of the year (Mascart et al. 2015).

The in situ experiment, comprised of cylindrical experimental PVC units (inner diameter of $10 \mathrm{~cm}$ ), was set up by scuba divers on 26 October 2012 for $24 \mathrm{~h}$ of incubation and on 2 November 2012 for $96 \mathrm{~h}$ of incubation. During the experiment, the site was characterized by a constant salinity of 38 , calm weather conditions and weak currents $\left(4-5 \mathrm{~cm} \mathrm{~s}^{-1}\right)$. Temperatures varied between 18 and $21{ }^{\circ} \mathrm{C}$ and light intensities were highest (1200-4000 lux) between 11 a.m. and 14 p.m. $\left(\mathrm{HOBO}^{\circledR}\right.$ Onset Computer Corporation).

The cylindrical experimental PVC units were divided into two parts: (1) an upper compartment containing on average $12 \pm 6$ gDW fresh macrophytodetritus (i.e. slightly degraded dead seagrass leaves) (MPD) and (2) a lower compartment containing sandy sediment (SED) (Fig. 1). The upper compartment had a height of $15 \mathrm{~cm}$, as used in emergence traps (Walters and Bell 1986) in order to exclude water flow-driven effects and random contamination of mesopsammic copepods. At $10 \mathrm{~cm}$ height, a circumference window was made and together with the open top of the tube, both were covered with a $38-\mu \mathrm{m}$ mesh, in order to exclude any contamination and predation by macrofauna 
Fig. 1 Experimental design representing the four treatments, showing the dimensions, the two mesh sizes used, the defaunated habitats and the two compartments: upper (on top of the sediments) and lower (inside the sediments). MPD macrophytodetritus. SED sediments, $W C$ water column
Watercolumn (WC)

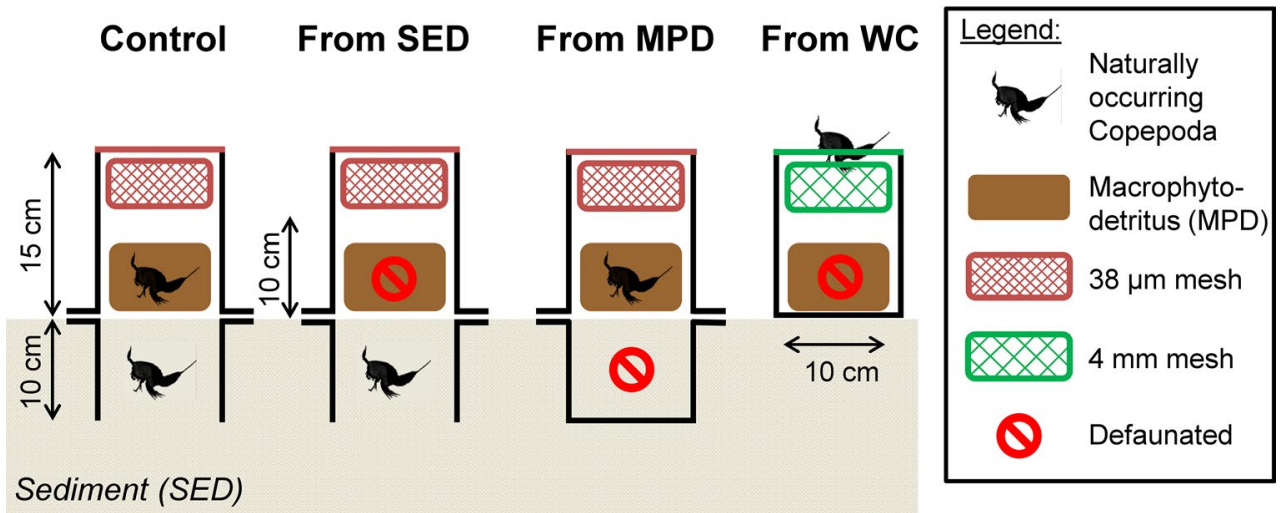

but to allow water and oxygen exchange. The lower compartment was inserted $10 \mathrm{~cm}$ deep into the sediment. This depth was chosen since vertical penetration of copepods happens in surficial layer (Danovaro and Fraschetti 2002; Kotwicki et al. 2005; Giere 2009), rarely exceeding 5-10 cm depth and therefore contamination from the surrounding sediments is excluded. The bottom of the tube remained open. Both compartments were placed on top of each other and fitted to stabilizing plates at the watersediment interface providing support. The stabilizing plates offered guidance for the insertion of the splitter plate used to collect upper or lower compartment at the end of the incubation (Fig. 1).

The experiment was conducted twice. A first short-term incubation of $24 \mathrm{~h}\left(T_{24}\right)$ lasting a full diurnal cycle was carried out to rule out any pattern of vertical migration due to the change in light intensity (Walters and Bell 1986). A second mid-term incubation of $96 \mathrm{~h}\left(T_{96}\right)$ was performed to give a chance to the colonizing community to stabilize, however, not too long to avoid potential intra-specific competition and thus a colonization-competition trade-off (Chandler and Fleeger 1983; De Troch et al. 2005). Both experiments started at midday, but were not set up simultaneously to avoid disturbance during collection of an experiment. Each experiment was preceded by a reference sampling $\left(T_{0}\right)$ in order to define the natural species composition and variability in the adjacent, potential source pool, namely the natural bare sandy sediments $\left(T_{0}\right.$ SED), the natural macrophytodetritus ( $T_{0}$ MPD), the adjacent $P$. oceanica canopy ( $\left.T_{0} \mathrm{POS}\right)$ and the water column $1 \mathrm{~m}$ above the site $\left(T_{0} \mathrm{WC}\right)$. These four habitats were sampled with, respectively, sediment meiocores (De Troch et al. 2001), detritus-cores (Mascart et al. 2015), plastic bags (Lepoint et al. 2006; Mascart et al. 2013) and 50- $\mu \mathrm{m}$-mesh hand towed horizontal plankton nets (Hamner and Carleton 1979), respectively.

The experimental design consisted of four treatments in quadruplicates (Fig. 1). The first treatment 'Control' was set to test for possible effects of the deployed units on meiofauna MPD and sediment natural community. The upper and lower compartments were filled with natural macrophytodetritus and sediments, respectively. The second treatment 'From SED' tested the colonization of copepods from natural bare sediments towards defaunated macrophytodetritus positioned above it. The upper and lower compartments were filled with defaunated macrophytodetritus and natural sediments, respectively. The third treatment 'From MPD' tested the opposite colonization direction from natural macrophytodetritus towards defaunated sediments. The upper compartment contained natural macrophytodetritus, while the lower compartment contained defaunated sediments. The lower compartment was closed to suppress colonization by the sediment. The forth treatment 'From WC' only consisted of an upper compartment filled with defaunated macrophytodetritus and was closed with a 4-mm mesh to allow larger planktonic copepods to enter the system, while excluding macrofaunal predators (e.g. juvenile fishes, amphipod crustaceans and shrimps). This last treatment tested for copepod colonization towards the macrophytodetritus from the surrounding water column.

Defaunation of macrophytodetritus and sediment was performed prior to the experiment. For this purpose, additional natural samples of macrophytodetritus were taken and were rinsed thoroughly with fresh water and an $8 \%$ $\mathrm{MgCl}_{2}$-solution (Hulings and Gray 1971) on a 1-mm sieve in order to stun and remove attached organisms, while keeping the loss of epiphytes living on the dead leaves surface as minimal as possible. Additional sediment samples were collected and defaunated by, a gentle defaunization technique (in contrast to the destructive methods applied by Chandler and Fleeger 1983; Chertoprud et al. 2005) to prevent loss of attractiveness for the potential colonizing copepods. The sediments were bathed in freshwater for several minutes (to detach copepods), mixed by hand and decanted. A control subsample was taken and analysed under a stereomicroscope to confirm the successful defaunation. The 
process was repeated on average five times until no copepods remained.

Sample collection and treatment

At the end of the incubation, prior to sampling, both compartments were isolated by inserting a splitter plate in between the stabilizing plates. Each compartment together with its content was subsequently transferred to a closed plastic zip bag to avoid contamination and any loss of material. The upper compartments containing macrophytodetritus were afterwards rinsed with an $8 \% \mathrm{MgCl}_{2}$-solution and fresh water in order to separate meiofauna from the macrophytodetritus. The rinsed samples were sieved over a 1-mm and 38- $\mu \mathrm{m}$-mesh sieve to exclude macrophytodetritus and retain copepods, respectively. The copepods were preserved in a $4 \%$ formaldehyde seawater solution. The separated macrophytodetritus was dried at $60{ }^{\circ} \mathrm{C}$ for 4 days (Mascart et al. 2015) to measure dry weight for further standardization of copepod densities. The lower compartments comprising sediments were subsampled with meiocores and afterwards preserved in a $4 \%$ formaldehyde seawater solution. The copepods present in the sediment were subsequently extracted by centrifugation with Ludox HS40 (specific density of $1.18 \mathrm{~g} \mathrm{dm}^{-3}$ ). The copepods were stained with Rose Bengal, counted, picked out and mounted on slides for microscopic identification to species level based on identification keys of Lang (1948) and Boxshall and Hasley (2004). Densities of copepods in the macrophytodetritus were standardized towards the dry weight of macrophytodetritus (indiv. $\mathrm{g}^{-1} \mathrm{DW}$ ) and towards the sediment surface in the case of benthic samples (indiv. $10 \mathrm{~cm}^{-2}$ ).

\section{Data analysis}

To analyse the structure of the community, five species diversity metrics were used reflecting the different elements of biodiversity (see Magurran (2004) and Magurran and McGill (2011) for an overview): $S=$ number of species observed (species number); $d=$ Margalef's corrected number of species for $N$ number of individuals (species richness); $H^{\prime}=$ the Shannon's diversity index based on natural logarithm (species diversity); $E_{\mathrm{H}}=$ Heip's evenness index sensitive to rare species (species evenness); and $N_{1}=$ number of species that would have been found in the sample when all species would be equally common (dominance metrics).

In order to identify the most typifying copepod species primarily providing the discrimination between and within factors, a SIMPER (similarity percentages) routine was used after an ANOSIM (analysis of similarity) difference test. To visualize the community structure a principal coordinate analysis (PCO) based on the zero-adjusted
Bray-Curtis similarity resemblance matrix of the log-transformed relative multivariate data of copepod species abundances was performed.

The analysis of variance in univariate or multivariate data were examined using a PERMANOVA routine and post hoc pairwise comparisons with fixed factors Treatment (Control, From SED, From MPD and From WC), Habitat (MPD, SED) or Time $\left(T_{0}, T_{24}\right.$ and $\left.T_{96}\right)$. PERMANOVA allows us to perform an ANOVA with $P$ values obtained by permutation (Anderson et al. 2008), thus avoiding the assumption of normality. Prior to run two-way PERMANOVA's, assumption of homogeneity of dispersion was tested with a PERMDISP and distances amongst centroids calculated on the interaction level (Quinn and Keough 2002). Euclidean distance and Bray-Curtis-based resemblance matrices were used, respectively, for univariate and multivariate measures. Pairwise tests type III and Monte-Carlo $P$ values were used since sometimes the total number of unique permutations did not exceed a hundred, whereas 4999 unique permutation is favourable (Anderson et al. 2008).

The calculated resemblance matrixes were based on a zero-adjusted log-transformed data with the addition of one dummy variable, for the reason that defaunated compartments contained no species at the start of the incubation. Prior to the analysis, both experiments' reference samples ( $T_{0}$ samples of $T_{24}$ and $T_{96}$ ) were checked for significant differences in species composition for the factors Habitat and Time. No differences were found between both time references sample for each habitat $(P=0.471)$. Within each duration, all four reference habitats were significantly different in species composition $(P<0.001)$. Therefore, both incubations reference samples were pooled into one $T_{0}$ reference per habitat in order to have a higher replication and thus higher statistical results. Due to different standardization methods within each unit compartment (habitat MPD per gram dry weight vs. SED per surface area), differences in total absolute copepod densities were examined per compartment using factors Time and Treatment. Variance in species compositions between the start $\left(T_{0}\right)$ and the end of incubation $\left(T_{24}\right.$ or $\left.T_{96}\right)$ were investigated using the fixed factors Treatment and Habitat.

All the above-mentioned analyses were performed with the Primer 6.1.11 software (Clarke and Gorley 2006) with PERMANOVA add-on software (Anderson et al. 2008). A significance level of $P<0.05$ was used in all tests. Graphs were constructed in GraphPad 5.03 for Windows (GraphPad Software, San Diego California USA).

\section{Results}

Within 24 and $96 \mathrm{~h}$ of incubation, all defaunated habitats were colonized by copepods; therefore, no repulsive effect 


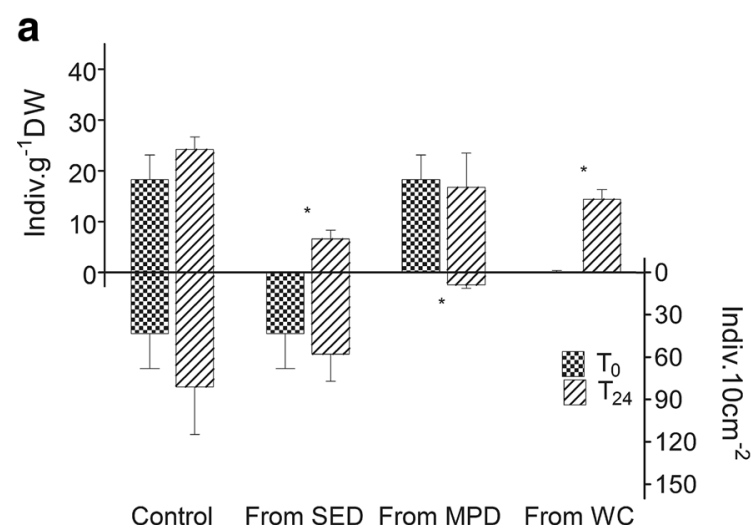

Fig. 2 Mean Copepoda densities per treatment for the 24-h incubation experiment (a) and the 96-h incubation experiment (b). Upper part represents the upper compartment with macrophytodetritus $(M P D)$ standardized per indiv. $\mathrm{g}^{-1} \mathrm{DW}$ (dry weight) on the left $y$ axis. Lower part represents the lower compartment with the sediments

of the experimental set-up was found (all pairwise $P>0.1$ ) (Fig. 2). Over all samples, a total of 58 different species were identified belonging to three Copepoda orders. The majority of the species (50) belonged to the order harpacticoida, representing $83.8 \pm 2.1 \%$ (average $\pm \mathrm{SD}$, henceforth used as notation) of the encountered species. Five species belonged to the order Cyclopoida and three species to the calanoida order (Table 1). Noodt (1971) attempted a provisional classification of Copepoda based on the variety of morphological forms which are adapted to special conditions in various habitats. All eco-morphological types except parasitic types were present in this study: (M) Mesopsammic types, primarily sediment living; (P) Phytal types, clinging to phytal structures; (E) Epibenthic types, benthic-swimmers; (W) Water column types, pelagic freeswimmers. A complete classification per species was made here (Table 1) in accordance with former studies and morpho-ecological traits (Lang 1948; Noodt 1971; Bell et al. 1987; Higgins and Thiel 1988; Bodin and Leguellec 1992; Thistle and Sedlacek 2004). Following the obtained results, a classification in terms of active colonization was added per species: (I) infaunal colonizers, interstitial dispersal pathway; (S) suspension colonizers, water-bound dispersal pathway; and (-) non-active colonizer, persists in its initial habitat (Table 1). In order to keep a comprehensive overview, the results are presented per factor Time and succinctly by factor Treatment.

\section{Reference samples $T_{0}$}

All $T_{0}$ reference samples in sediment, water column, $P$. oceanica canopy and macrophytodetritus were significantly different from each other in terms of species composition (ANOSIM, global $R=0.994, P<0.001$ ). The

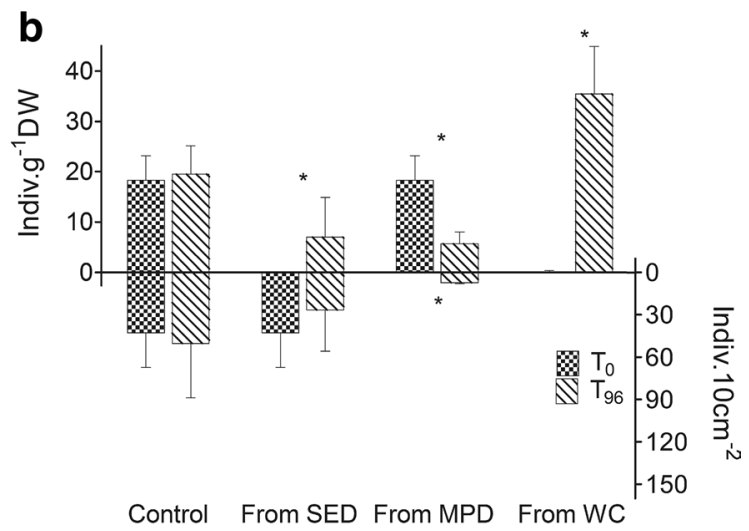

(SED) standardized per indiv. $10 \mathrm{~cm}^{-2}$ on the right $y$ axis. Error bars represent the standard deviation. $N=4$ per treatment and $W C$ water column. Asterisk shows a significant difference $(P<0.05)$ in total densities between start and end incubation

top four species typifying a reference habitat were always distinct with the exception of Sarsamphiascus tenuiremis (Miraciidae family) which is omnipresent in all reference habitats, except for the water column (Table 2). Since a high similarity between replicates was found in each habitat, the dissimilarity between pairs of habitats is as expected high $(>81 \%)$, except between the macrophytodetritus and $P$. oceanica canopy where the dissimilarity is reduced to $46.2 \%$. The average evenness $\mathrm{E}_{\mathrm{H}}$ was $0.27 \pm 0.04$ for all samples. The highest number of species and species richness was accounted for macrophytodetritus with an $S$ of $25.3 \pm 1.0$ and $d=5.1 \pm 0.2$, which is quite similar to the number of species in the $P$. oceanica canopy with $S=23.5 \pm 1.7$ and $d=4.9 \pm 0.3$. The sediments had a lower number of species $S=14.3 \pm 0.6$ and richness $d=3.6 \pm 0.3$. The lowest species number and richness was found in the water column with $S=8.0 \pm 1.4$ and $d=1.6 \pm 0.3$. The evenness was similar in all four samples. The total copepod density was $42.7 \pm 24.5$ indiv. $10 \mathrm{~cm}^{-2}$ for the bare sediments $\left(T_{0}\right.$ SED), $18.3 \pm 4.9$ indiv. $\mathrm{g}^{-1}$ DW for the natural macrophytodetritus $\left(T_{0}\right.$ MPD), $8.1 \pm 2.4$ indiv. $\mathrm{g}^{-1}$ DW for the $P$. oceanica canopy ( $T_{0}$ POS) and $120.2 \pm 4.6$ indiv. $\mathrm{m}^{-3}$ for the water column ( $\left.T_{0} \mathrm{WC}\right)$.

All the species found in the seagrass meadow were found in macrophytodetritus, with the exception of Sacodiscus littoralis (family Tisbidae). It was the only noncolonizing species exclusively found in the $P$. oceanica canopy. The species Ambunguipes rufocincta, Probosciphontodes stellate, Syngastes cornalinus, Tegastes calcaratus, Rhynchothalestris helgolandica and Xouthous laticaudatus were only present in the macrophytodetritus habitat, while the following species were exclusively present in the sediment: Arenosetella tenuissima, Canuella 
Table 1 Cumulative presence list of Copepoda species sorted per order and per family based on four replicates

\begin{tabular}{|c|c|c|c|c|c|c|c|c|c|c|c|c|c|c|c|c|c|}
\hline & & & & & $\mathrm{T}_{0}$ & & & & & $\mathrm{~T}_{2}$ & 24 & & & & & & $\mathrm{~T}_{96}$ \\
\hline Active & Ecologic. & Treatment: & & & & & Cor & introl & From & $\mathrm{mSED}$ & From & m MPD & From WC & & ntrol & & $\mathrm{m} \mathrm{SED}$ \\
\hline Colonizer & Type & Habitat: & SED & MPD & POS & WC & MPD & SED & & & & & & & SED & & D SED \\
\hline & & Harpacticoida & & & & & & & & & & & & & & & \\
\hline & & Ameiridae & & & & & & & & & & & & & & & \\
\hline I/S & M & Ameira longipes (Boeck, 1865) & & $\mathrm{x}$ & $\mathrm{x}$ & $\mathrm{x}$ & $\mathrm{x}$ & $\mathrm{x}$ & $\mathrm{x}$ & & $\mathrm{x}$ & $\mathrm{x}$ & $\mathrm{x}$ & $\mathrm{x}$ & $\mathrm{x}$ & $\mathrm{x}$ & \\
\hline $\mathrm{s}$ & M & Ameiropsis nobilis (Sars G.O., 1911) & $\mathrm{x}$ & $\mathrm{x}$ & & & $\mathrm{x}$ & & & $\mathrm{X}$ & $\mathrm{x}$ & & $\mathrm{x}$ & $\mathrm{x}$ & & & \\
\hline IS & $P$ & Ancorabolidae & & & & & & & & & & & & & & & \\
\hline $\begin{array}{c}\mathrm{I} / \mathrm{S} \\
-\end{array}$ & $\begin{array}{l}P \\
P\end{array}$ & $\begin{array}{l}\text { Laophontodes bicornis (Scott A., 1896) } \\
\text { Probosciphontodes stellata (Fiers, 1988) }\end{array}$ & & $\mathrm{x}$ & $\mathrm{x}$ & & $\mathrm{X}$ & & & & & & $\mathrm{x}$ & $\mathrm{X}$ & & $\mathrm{x}$ & \\
\hline - & & $\begin{array}{l}\text { Probosciphontodes stellata (Fiers, 1988) } \\
\text { Canuelidae }\end{array}$ & & $\mathrm{X}$ & & & $\mathrm{x}$ & & & & & & & $\mathrm{x}$ & & & \\
\hline- & M & $\begin{array}{l}\text { Canuella furcigera (Sars G.O., 1903) } \\
\text { Cletodidae }\end{array}$ & $\mathrm{x}$ & & & & & $\mathrm{x}$ & & $\mathrm{x}$ & & & & & $\mathrm{x}$ & & $\mathrm{x}$ \\
\hline $\mathrm{I} / \mathrm{S}$ & M & Cletodes limicola (Brady, 1872) & $\mathrm{x}$ & $\mathrm{x}$ & & & & & & $\mathrm{x}$ & & $\mathrm{x}$ & $\mathrm{x}$ & & & & \\
\hline $\mathrm{s}$ & $\mathrm{P}$ & Dactylopusia tisboides (Claus, 1863) & & $\mathrm{X}$ & $\mathrm{x}$ & & $\mathrm{x}$ & & & & $\mathrm{X}$ & & $\mathrm{x}$ & $\mathrm{x}$ & & & \\
\hline - & $\mathrm{P}$ & Diarthrodes minutus (Claus, 1863) & & $\mathrm{x}$ & $\mathrm{x}$ & & $\mathrm{x}$ & & & & $\hat{x}$ & & & $\mathrm{x}$ & & & \\
\hline - & $\mathrm{P}$ & Paradactylopodia brevicornis (Claus, 1866) & & $\mathrm{x}$ & $\mathrm{x}$ & & $\mathrm{x}$ & & & & $\mathrm{x}$ & & & $\mathrm{x}$ & & & \\
\hline & & Ectinosomatidae & & & & & & & & & & & & & & & \\
\hline $\mathrm{I} / \mathrm{S}$ & M & Ectinosoma dentatum (Steuer, 1940) & $\mathrm{x}$ & $\mathrm{x}$ & & & $\mathrm{x}$ & $\mathrm{x}$ & $\mathrm{X}$ & $\mathrm{x}$ & $\mathrm{x}$ & $\mathrm{x}$ & $\mathrm{x}$ & $\mathrm{x}$ & $\mathrm{x}$ & $\mathrm{x}$ & $\mathrm{x}$ \\
\hline- & M & Arenosetella tenuissima (Klie, 1929) & $\mathrm{x}$ & & & & & & & $\mathrm{x}$ & & & & & & & $\mathrm{x}$ \\
\hline - & M & Pseudobradya hirsuta (Scott T. \& A., 1896) & $\mathrm{X}$ & & & & & & & $\mathrm{x}$ & & & & & & & $\mathrm{x}$ \\
\hline $\mathrm{s}$ & $\mathrm{W}$ & Microsetella norvegica (Boeck, 1865) & & & & $\mathrm{x}$ & & & & & & & $\mathrm{x}$ & & & & \\
\hline & & Hamondidae & & & & & & & & & & & & & & & \\
\hline - & $\mathrm{P}$ & Ambunguipes rufocincta (Norman in Brady, 1880) & & $\mathrm{x}$ & & & $\mathrm{x}$ & & & & $\mathrm{x}$ & & & $\mathrm{x}$ & & & \\
\hline $\mathrm{s}$ & $P$ & $\begin{array}{l}\text { Harpacticidae } \\
\text { Harpacticus littoralis (Sars G.O., 1910) }\end{array}$ & & $\mathrm{x}$ & $\mathrm{x}$ & & $\mathrm{x}$ & & & & $\mathrm{x}$ & & & & & & \\
\hline I & M & $\begin{array}{l}\text { Laophontidae } \\
\text { Asellopsis duboscqui (Monard, 1926) }\end{array}$ & $\mathrm{x}$ & $\mathrm{x}$ & & & & & $\mathrm{x}$ & $\mathrm{x}$ & $\mathrm{x}$ & $\mathrm{x}$ & & & & $\mathrm{x}$ & \\
\hline $\mathrm{s}$ & $\mathrm{P}$ & Esola longicauda (Edwards, 1891) & & & & & & & & & & & $\mathrm{x}$ & & & & \\
\hline I & $\mathrm{M}$ & Laophonte cornuta (Philippi, 1840) & $\mathrm{x}$ & & & & $\mathrm{x}$ & & $\mathrm{x}$ & & $\mathrm{x}$ & & & $\mathrm{x}$ & & & \\
\hline $\mathrm{I} / \mathrm{S}$ & M & Laophonte elongata elongata (Boeck, 1873) & $\mathrm{X}$ & $\mathrm{x}$ & & & $\mathrm{x}$ & & $\mathrm{X}$ & & & & $\mathrm{x}$ & $\mathrm{x}$ & & $\mathrm{x}$ & \\
\hline I & M & Laophontina posidoniae (Fiers, 1986) & $\mathrm{x}$ & & & & & $\mathrm{x}$ & $\mathrm{x}$ & $\mathrm{x}$ & & & & & $\mathrm{x}$ & & $\mathrm{x}$ \\
\hline $\mathrm{I} / \mathrm{S}$ & $\mathrm{P}$ & Paralaophonte brevirostris (Claus, 1863) & & $\mathrm{x}$ & $\mathrm{x}$ & & $\mathrm{x}$ & & $\mathrm{x}$ & & $\mathrm{x}$ & $\mathrm{x}$ & $\mathrm{x}$ & $\mathrm{x}$ & & $\mathrm{x}$ & \\
\hline I & M & $\begin{array}{l}\text { Leptastacidae } \\
\text { Leptastacus laticaudatus (Nicholls, 1935) }\end{array}$ & $\mathrm{x}$ & & & & & $\mathrm{x}$ & & $\mathrm{x}$ & & $\mathrm{x}$ & & & $\mathrm{x}$ & & $\mathrm{x}$ \\
\hline & & Leptopontiidae & & & & & & & & & & & & & & & \\
\hline I & M & Leptopontia curvicauda (Scott,1902) & $\mathrm{x}$ & $\mathrm{x}$ & & & & $\mathrm{x}$ & $\mathrm{x}$ & $\mathrm{x}$ & & $\mathrm{x}$ & & & $\mathrm{x}$ & & $\mathrm{x}$ \\
\hline $\mathrm{s}$ & $P$ & $\begin{array}{l}\text { Longipedididae } \\
\text { Longipedia minor (Scott T. \& A., 1893) } \\
\text { Metidae }\end{array}$ & & $\mathrm{x}$ & $\mathrm{x}$ & & $\mathrm{x}$ & & & & $\mathrm{x}$ & & $\mathrm{x}$ & $\mathrm{x}$ & & $\mathrm{x}$ & \\
\hline I & $\mathbf{P}$ & Metis ignea (Philippi, 1843) & & $\mathrm{x}$ & $\mathrm{x}$ & & $\mathrm{x}$ & $\mathrm{x}$ & $\mathrm{x}$ & & $\mathrm{x}$ & $\mathrm{x}$ & & $\mathrm{x}$ & & & \\
\hline JS & $M$ & Miraciidae & & & & & & & & & & & & & & & \\
\hline $\mathrm{I} / \mathrm{S}$ & $\mathrm{M}$ & Amphiascoides debilis (Giesbrecht, 1881) & $\mathrm{X}$ & $\mathrm{X}$ & $\mathrm{X}$ & & $\mathrm{X}$ & $\mathrm{X}$ & $\mathrm{X}$ & $\mathrm{X}$ & $\mathrm{X}$ & $\mathrm{x}$ & $\mathrm{X}$ & $\mathrm{X}$ & $\mathrm{X}$ & $\mathrm{X}$ & $\mathrm{X}$ \\
\hline $\mathrm{I} / \mathrm{S}$ & $\mathrm{P}$ & Diosaccus temuicornis (Claus, 1863) & $\mathrm{X}$ & $\mathrm{X}$ & $\mathrm{X}$ & & $\mathrm{X}$ & $\mathrm{x}$ & $\mathrm{X}$ & $\mathrm{x}$ & $\mathrm{x}$ & & $\mathrm{x}$ & $\mathrm{x}$ & $\mathrm{X}$ & $\mathrm{x}$ & $\mathrm{X}$ \\
\hline I/S & $\mathrm{M}$ & Amphiascus minutus (Claus, 1863) & $\mathrm{x}$ & $\mathrm{x}$ & $\mathrm{x}$ & & $\mathrm{x}$ & $\mathrm{x}$ & $\mathrm{x}$ & & $\mathrm{x}$ & $\mathrm{x}$ & $\mathrm{x}$ & $\mathrm{x}$ & $\mathrm{X}$ & $\mathrm{x}$ & $\mathrm{x}$ \\
\hline I/S & M & Sarsamphiascus temuiremis (Brady, 1880) & $\mathrm{x}$ & $\mathrm{x}$ & $\mathrm{x}$ & & $\mathrm{x}$ & $\mathrm{x}$ & $\mathrm{x}$ & $\mathrm{x}$ & $\mathrm{x}$ & $\mathrm{x}$ & $\mathrm{x}$ & $\mathrm{x}$ & $\mathrm{x}$ & $\mathrm{x}$ & $\mathrm{x}$ \\
\hline $\mathrm{I} / \mathrm{S}$ & M & Delavalia normani (Scott T., 1905) & $\mathrm{x}$ & $\mathrm{x}$ & & & $\mathrm{x}$ & $\mathrm{x}$ & $\mathrm{x}$ & $\mathrm{x}$ & $\mathrm{x}$ & $\mathrm{x}$ & $\mathrm{x}$ & & $\mathrm{x}$ & & \\
\hline & & Rhizotrichidae & & & & & & & & & & & & & & & \\
\hline I & M & Rhizothrix curvatum (Brady, 1880) & $\mathrm{x}$ & $\mathrm{x}$ & & & & $\mathrm{x}$ & & $\mathrm{x}$ & & $\mathrm{x}$ & & & $\mathrm{x}$ & & $\mathrm{x}$ \\
\hline & & Paramesochridae & & & & & & & & & & & & & & & \\
\hline I & $\mathrm{M}$ & Wellsopsyllus (Scott.) robertsoni (Scott T. \& A., 1895) & $\mathrm{x}$ & $\mathrm{x}$ & & & & $\mathrm{x}$ & & $\mathrm{x}$ & $\mathrm{x}$ & $\mathrm{x}$ & & & $\mathrm{x}$ & & $\mathrm{x}$ \\
\hline I & M & Wellsopsyllus (Inter.) intermedius (Scott T. \& A, 1895) & $\mathrm{x}$ & & & & & $\mathrm{x}$ & & & & $\mathrm{x}$ & & & $\mathrm{x}$ & & \\
\hline $\mathrm{s}$ & $\mathrm{P}$ & $\begin{array}{l}\text { Peltiidae } \\
\text { Alteutha depressa (Claus, 1863) }\end{array}$ & & $\mathrm{x}$ & $\mathrm{x}$ & & $\mathrm{x}$ & & & & $\mathrm{x}$ & & $\mathrm{x}$ & $\mathrm{x}$ & & & \\
\hline & & Porcellididae & & & & & & & & & & & & & & & \\
\hline $\mathrm{s}$ & $\mathrm{P}$ & Porcellidium ovatum (Haller, 1879) & & $\mathrm{x}$ & $\mathrm{x}$ & & $\mathrm{x}$ & & & & $\mathrm{x}$ & & $\mathrm{x}$ & $\mathrm{x}$ & & & \\
\hline $\mathrm{s}$ & $\mathrm{P}$ & Porcellidium fimbriatum (Claus, 1863) & & $\mathrm{x}$ & $\mathrm{x}$ & & $\mathrm{x}$ & & & & $\mathrm{x}$ & & & $\mathrm{x}$ & & & \\
\hline & & Pseudotachidiidae & & & & & & & & & & & & & & & \\
\hline $\mathrm{I} / \mathrm{S}$ & $\mathrm{P}$ & Dactylopodella flava (Claus, 1866) & & $\mathrm{x}$ & $\mathrm{x}$ & & $\mathrm{x}$ & $\mathrm{x}$ & $\mathrm{x}$ & $\mathrm{x}$ & $\mathrm{x}$ & $\mathrm{x}$ & $\mathrm{x}$ & $\mathrm{x}$ & & $\mathrm{x}$ & \\
\hline $\mathrm{s}$ & $\mathrm{P}$ & Xouthous laticaudatus (Thompson I.C. \& Scott A., 1903) & & $\mathrm{x}$ & & & & & & & & & & $\mathrm{x}$ & & $\mathrm{x}$ & \\
\hline & & Tegastidae & & & & & & & & & & & & & & & \\
\hline $\mathrm{s}$ & $\mathrm{P}$ & Parategastes sphaericus (Claus, 1863) & & & $\mathrm{x}$ & & & & & & $\mathrm{X}$ & & $\mathrm{x}$ & & & & \\
\hline $\mathrm{s}$ & $\mathrm{P}$ & Syngastes cornalinus (Monard, 1924) & & $\mathrm{x}$ & & & $\mathrm{x}$ & & & & $\mathrm{x}$ & & $\mathrm{x}$ & & & & \\
\hline - & $\mathrm{P}$ & Tegastes calcaratus (Sars G.O., 1910) & & $\mathrm{x}$ & & & & & & & $\mathrm{x}$ & & & & & & \\
\hline $\mathrm{s}$ & $\mathrm{P}$ & Tegastes satyrus (Claus, 1860) & & $\mathrm{x}$ & $\mathrm{x}$ & & $\mathrm{x}$ & & & & $\mathrm{x}$ & & $\mathrm{x}$ & $\mathrm{x}$ & & $\mathrm{x}$ & \\
\hline & & Tetragonicepsidae & & & & & & & & & & & & & & & \\
\hline $\mathrm{I} / \mathrm{S}$ & $\mathrm{P}$ & Diagoniceps laevis (Willey, 1930) & & & $\mathrm{x}$ & & $\mathrm{x}$ & $\mathrm{x}$ & & & & & $\mathrm{x}$ & & $\mathrm{x}$ & & \\
\hline I/S & M & Phyllopodopsyllus bradyi (Scott T., 1892) & $\mathrm{x}$ & $\mathrm{x}$ & & & & & & $\mathrm{x}$ & $\mathrm{x}$ & $\mathrm{x}$ & $\hat{x}$ & $\mathrm{x}$ & $\mathrm{X}$ & & $\mathrm{x}$ \\
\hline I & M & Tetragoniceps scotti (Sars G.O., 1911) & $\mathrm{x}$ & & & & & $\mathrm{x}$ & & & & $\mathrm{x}$ & & & $\mathrm{x}$ & & \\
\hline & & Thalestridae & & & & & & & & & & & & & & & \\
\hline $\mathrm{s}$ & $P$ & $\begin{array}{l}\text { Rhynchothalestris helgolandica (Claus, 1863) } \\
\text { Tisbidae }\end{array}$ & & $\mathrm{X}$ & & & & & & & $\mathrm{x}$ & & & $\mathrm{x}$ & & & \\
\hline $\mathrm{s}$ & $\mathrm{E}$ & $\begin{array}{l}\text { Tisbidae } \\
\text { Tisbe elegantula (Sars G.O., 1905) }\end{array}$ & & $\mathrm{x}$ & $\mathrm{x}$ & & $\mathrm{x}$ & & & & $\mathrm{x}$ & & $\mathrm{x}$ & $\mathrm{x}$ & & & \\
\hline $\mathrm{s}$ & $\mathrm{E}$ & Tisbe ensifer (Fischer, 1860) & & $\hat{x}$ & $\mathrm{x}$ & & $\mathrm{x}$ & & & & & & $\hat{x}$ & & & & \\
\hline $\mathrm{s}$ & $\mathrm{E}$ & Tisbe furcata (Baird, 1837) & & $\mathrm{x}$ & $\mathrm{x}$ & & $\mathrm{x}$ & $\mathrm{x}$ & $\mathrm{x}$ & & $\mathrm{x}$ & & $\mathrm{x}$ & $\mathrm{x}$ & & $\mathrm{x}$ & $\mathrm{x}$ \\
\hline - & $\mathrm{P}$ & Sacodiscus littoralis (Sars G.O., 1904) & & & $\mathrm{x}$ & & & & & & & & & & & & \\
\hline & & $\begin{array}{l}\text { Calanoida } \\
\text { Clausocalanidae }\end{array}$ & & & & & & & & & & & & & & & \\
\hline $\mathrm{s}$ & w & Clausocalanus arcuicornis (Dana, 1849) & & $\mathrm{x}$ & $\mathrm{x}$ & $\mathrm{x}$ & $\mathrm{x}$ & $\mathrm{x}$ & $\mathrm{x}$ & $\mathrm{x}$ & $\mathrm{x}$ & $\mathrm{x}$ & $\mathrm{x}$ & $\mathrm{x}$ & & & \\
\hline & & Lucicutiidae & & & & & & & & & & & & & & & \\
\hline $\mathrm{s}$ & $\mathrm{w}$ & Lucicutia magna (Wolfenden, 1903) & & & $\mathrm{x}$ & $\mathrm{x}$ & $\mathrm{x}$ & $\mathrm{x}$ & & & & & $\mathrm{x}$ & $\mathrm{x}$ & & $\mathrm{x}$ & \\
\hline & & Paracalanidae & & & & & & & & & & & & & & & \\
\hline - & W & Paracalanus parvus parvus (Claus, 1863) & & & & $\mathrm{x}$ & & & & & & & & & & & \\
\hline & & Cyclopoida & & & & & & & & & & & & & & & \\
\hline & & Cyclopinidae & & & & & & & & & & & & & & & \\
\hline $\mathrm{s}$ & w & species 1 & $\mathrm{x}$ & $\mathrm{x}$ & $\mathrm{x}$ & $\mathrm{x}$ & $\mathrm{x}$ & $\mathrm{x}$ & $\mathrm{x}$ & & $\mathrm{x}$ & & $\mathrm{x}$ & $\mathrm{x}$ & $\mathrm{x}$ & & \\
\hline $\mathrm{s}$ & $\mathrm{w}$ & species 2 & & & & $\mathrm{x}$ & & & & & & & $\mathrm{x}$ & & & $\mathrm{x}$ & \\
\hline - & W & species 3 & & & & $\mathrm{x}$ & & & & & & & & & & & \\
\hline & & Oithonidae & & & & & & & & & & & & & & & \\
\hline $\mathrm{s}$ & w & Oithona nana (Giesbrecht, 1893) & & & $\mathrm{x}$ & $\mathrm{x}$ & & & & & & & & $\mathrm{x}$ & & $\mathrm{x}$ & $\mathrm{x}$ \\
\hline $\mathrm{s}$ & W & Oithona similis (Claus, 1866) & & & $\mathrm{x}$ & $\mathrm{x}$ & & & & & & & $\mathrm{x}$ & $\mathrm{x}$ & & $\mathrm{x}$ & \\
\hline
\end{tabular}

$\mathrm{X}=$ presence. Blank cells $=$ absence. The active colonization pathway in the outer left column are $I$ infaunal colonizers, interstitial dispersal pathway, $S$ suspension colonizers, water-bound dispersal pathway, - non-active colonizer, persists in its initial habitat. The ecological types presented in the second column are $M$ mesopsammic types, primarily sediment living, $P$ phytal types, clinging to phytal structures, $E$ epibenthic types, benthic-swimmers, $W$ water column types, pelagic free-swimmers 
Table 2 Multivariate PERMANOVA and SIMPER results representing the typifying species of the reference samples

\begin{tabular}{|c|c|c|}
\hline \multicolumn{3}{|l|}{ Species typifying reference habitats } \\
\hline Species & $\%$ & cum. $\%$ \\
\hline \multicolumn{3}{|l|}{$T_{0}$ SED ( $54.4 \%$ similarity) } \\
\hline Sarsamphiascus tenuiremis & 43.0 & 43.0 \\
\hline Leptastacus laticaudatus & 11.6 & 54.6 \\
\hline Wellsopsyllus (Scottopsyllus) robertsoni & 8.1 & 62.7 \\
\hline Amphiascoides debilis & 8.1 & 70.8 \\
\hline Ectinosoma dentatum & 8.0 & 78.7 \\
\hline \multicolumn{3}{|l|}{$T_{0}$ MPD (74.4 \% similarity) } \\
\hline Dactylopodella flava & 22.9 & 22.9 \\
\hline Ameira longipes & 12.3 & 35.2 \\
\hline Dactylopusia tisboides & 9.0 & 44.2 \\
\hline Sarsamphiascus tenuiremis & 8.0 & 52.2 \\
\hline Amphiascus minutus & 6.7 & 58.9 \\
\hline \multicolumn{3}{|l|}{$T_{0}$ POS (73.1 \% similarity) } \\
\hline Harpacticus littoralis & 14.3 & 14.3 \\
\hline Diosaccus tenuicornis & 12.2 & 26.5 \\
\hline Sarsamphiascus tenuiremis & 9.6 & 36.1 \\
\hline Amphiascus minutus & 7.6 & 43.6 \\
\hline Porcellidium ovatum & 6.4 & 50.0 \\
\hline \multicolumn{3}{|l|}{$T_{0} \mathrm{WC}(90.0 \%$ similarity $)$} \\
\hline Paracalanus parvus parvus & 36.3 & 36.3 \\
\hline Clausocalanus arcuicornis & 27.2 & 63.5 \\
\hline Oithona similis & 13.3 & 76.8 \\
\hline Cyclopinidae sp. 1 & 9.7 & 86.5 \\
\hline Microsetella norvegica & 7.8 & 94.3 \\
\hline
\end{tabular}

$T_{0}$ first five contributing species are shown, $S E D$ sediments, $M P D$ macrophytodetritus, POS Posidonia oceanica canopy, WC water column

furciger, Pseudobradya hirsuta and Wellsopsyllus (Intermediopsyllus) intermedius. All the water column habitat species were present in the newly colonized macrophytodetritus, except for Cyclopinidae sp. 3 and Paracalanus parvus parvus (Table 1 ).

\section{The 24-h experiment}

In the first experiment after an incubation of $24 \mathrm{~h}$, all defaunated habitats were colonized independently of the treatment (Fig. 2). The multivariate two-way PERMANOVA was significant for both factors (Time and Habitat) and interaction factor (Table 3). PERMDISP's for the interaction factor turned out to be significantly different, indicating that the variation within all factors and interactions was due to the dispersion and location effect, mainly because of the large number of zeros present. Pairwise comparisons revealed no significance difference between the $T_{24}$ 'Control' habitats and the $T_{0}$ reference samples (Table 3). In comparison with other treatments, the 'Control' treatment showed only significant differences for the treatment 'From SED' SED habitat (Pairwise: $t=2.39$, $P_{(\mathrm{MC})}=0.013$ ) and for 'From WC' MPD habitat (Pairwise: $\left.t=2.94, P_{(\mathrm{MC})}=0.003\right)($ Table 3$)$.

In the 'From SED' treatment, the defaunated MPD compartment was colonized by sediment copepods reaching a density of $6.6 \pm 1.7$ indiv. $\mathrm{g}^{-1}$ DW (Fig. 2a). A dissimilarity of $65.0 \%$ (SIMPER) was found between the 'From SED' and 'Control' treatment MPD habitat, although species composition was not significantly different (Table 3). In the 'From SED' treatment, the PCO clearly separates SED from MPD habitats, each were, however, similar at $65 \%$ (Fig. 3a). Diversity in this treatment is lower than the 'Control' sample (Table 5) due to the high level of dominance (>56\%) of S. tenuiremis (Table 4). The source pool community for this colonization, the SED habitat of the 'From SED' treatment had a lower diversity, evenness (Table 5) and a distinct species assemblage (Fig. 3a). Even though Sarsamphiascus tenuiremis remained the dominant species (Table 4).

The reverse treatment ('From MPD') tested a possible active migration from the natural macrophytodetritus to the defaunated sediments. The defaunated SED habitat was colonized with a density of $8.6 \pm 2.4$ indiv. $10 \mathrm{~cm}^{-2}$ and reached a diversity comparable to the 'Control' SED habitat. The PCO displayed an assemblage almost identical to the 'Control'. No significant difference in composition was found and four species of the top five most contributing species to the similarity were identical. $S$. tenuiremis was the dominant species (Table 4). The species assemblage had a low dissimilarity of $37.2 \%$ and Dactylopodella flava remained the dominant species (Table 4). The emigration to the sediments from the MPD compartment showed no significant impact on the latter's initial density (Fig. 2a) and diversity (Table 5), displaying the high variability present within the source pool.

Colonization from the surrounding water column was unambiguous since the density raised from zero to $14.4 \pm 1.9$ indiv. $g^{-1}$ DW (Fig. 2a). The species composition was dominated by a Cyclopoid species from the Cyclopinidae family and the calanoida, Clausocalanus arcuicornis (Table 4). The diversity metrics were in the same order of magnitude as in the 'Control' MPD habitat; however, the diversity and evenness were very low (Table 5). In comparison with treatment 'From SED', where the sediments exclusively serve as source pool a higher influx of individuals and species occurred. The species composition present after MPD colonization through the water column was significantly different from all four adjacent reference habitat samples (Table 3). 
Table 3 Two-way multivariate PERMANOVA of species composition and post hoc pairwise tests

\begin{tabular}{|c|c|c|c|c|}
\hline Factors and interaction & \multicolumn{2}{|c|}{$T_{24}$} & \multicolumn{2}{|r|}{$T_{96}$} \\
\hline Habitat (Ha) & & ${ }_{(1,2)}^{\prime}=12.9 ; P=0.001 * *$ & & $F_{(1,2)}^{\prime}=18.4 ; P=0.001 * *$ \\
\hline Treatment (Tr) & & ${ }_{(3,2)}^{\prime}=4.1 ; P=0.001 * *$ & & $F_{(3,2)}^{\prime}=3.2 ; P=0.001 * *$ \\
\hline $\mathrm{Ha} \times \mathrm{Tr}$ & & ${ }_{(3,2)}^{\prime}=2.5 ; P=0.011^{*}$ & & $F_{(3,2)}^{\prime}=2.8 ; P=0.003 * *$ \\
\hline \multirow[t]{2}{*}{ Pairwise comparisons } & \multicolumn{2}{|l|}{$T_{24}$} & \multicolumn{2}{|l|}{$T_{96}$} \\
\hline & SED & MPD & SED & MPD \\
\hline$T_{0}$ versus control & $t=1.39 ; P_{(\mathrm{MC})}=0.137$ & $t=1.30 ; P_{(\mathrm{MC})}=0.166$ & $t=1.97 ; P_{(\mathrm{MC})}=0.035^{*}$ & $t=2.09 ; P_{(\mathrm{MC})}=0.012 *$ \\
\hline From SED versus control & $t=2.39 ; P_{(\mathrm{MC})}=0.013^{*}$ & $t=1.69 ; P_{(\mathrm{MC})}=0.065$ & $t=1.86 ; P_{(\mathrm{MC})}=0.039^{*}$ & $t=1.61 ; P_{(\mathrm{MC})}=0.063$ \\
\hline From MPD versus control & $t=0.98 ; P_{(\mathrm{MC})}=0.431$ & $t=1.11 ; P_{(\mathrm{MC})}=0.293$ & $t=2.98 ; P_{(\mathrm{MC})}=0.004 * *$ & $t=1.30 ; P_{(\mathrm{MC})}=0.169$ \\
\hline From WC versus control & - & $t=2.94 ; P_{(\mathrm{MC})}=0.003^{* *}$ & - & $t=2.01 ; P_{(\mathrm{MC})}=0.011^{*}$ \\
\hline$T_{0} \mathrm{SED}$ versus from $\mathrm{WC}$ & - & $t=4.06 ; P=0.038^{*}$ & - & $t=5.13 ; P=0.032^{*}$ \\
\hline$T_{0} \mathrm{MPD}$ versus from $\mathrm{WC}$ & - & $t=3.48 ; P=0.031^{*}$ & - & $t=5.60 ; P=0.035^{*}$ \\
\hline$T_{0}$ POS versus from WC & - & $t=3.55 ; P=0.029^{*}$ & - & $t=5.36 ; P=0.032^{*}$ \\
\hline$T_{0} \mathrm{WC}$ versus from $\mathrm{WC}$ & - & $t=6.60 ; P=0.020^{*}$ & - & $t=8.77 ; P=0.033^{*}$ \\
\hline
\end{tabular}

$F^{\prime}$ pseudo- $F$ value, $P_{(\mathrm{MC})}$ Monte-Carlo $P$ value, $* 0.05>P>0.01=$ significant; $* * 0.01>P>0.001=$ highly significant. On the left side, the 24-h incubation experiment and on the right the 96-h incubation experiment

SED sediments, MPD macrophytodetritus, POS Posidonia oceanica canopy, WC water column

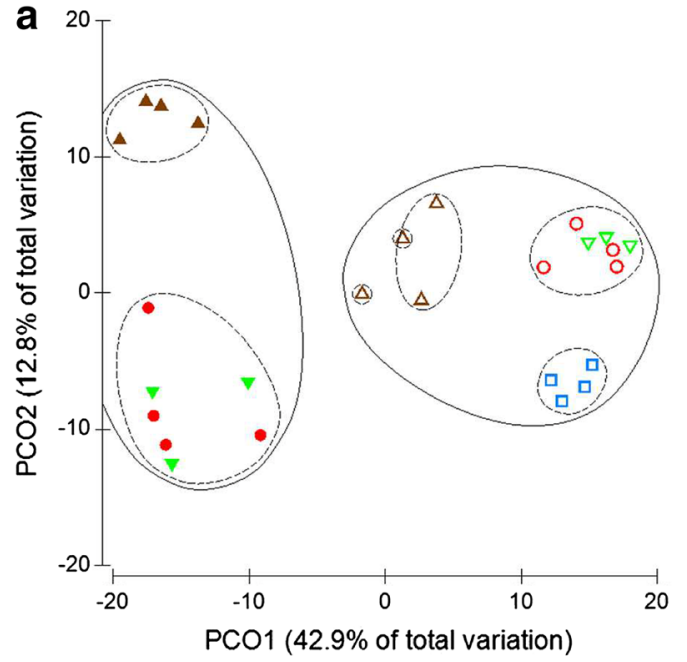

Fig. 3 Principal coordinate analysis (PCO) based on a Bray-Curtis similarity resemblance matrix on log-transformed data of species abundance of (a) 24-h incubation experiment and (b) 96-h incubation experiment. Filled symbols represent the sediments (SED) habitat and the un-filled symbols the macrophytodetritus (MPD) habitat. Differ-

\section{The 96-h experiment}

The copepod density in both 'Control' compartments of $\mathrm{T}_{96}$ was not significantly different from the respective $T_{0}$ reference samples habitat. A density of $19.5 \pm 5.7$ indiv. $\mathrm{g}^{-1} \mathrm{DW}$ and $50.3 \pm 38.4$ indiv. $10 \mathrm{~cm}^{-2}$ was, respectively, found for the 'Control' MPD and SED compartment (Fig. 2b). The diversity (S) was significantly lower (Table 5) in both

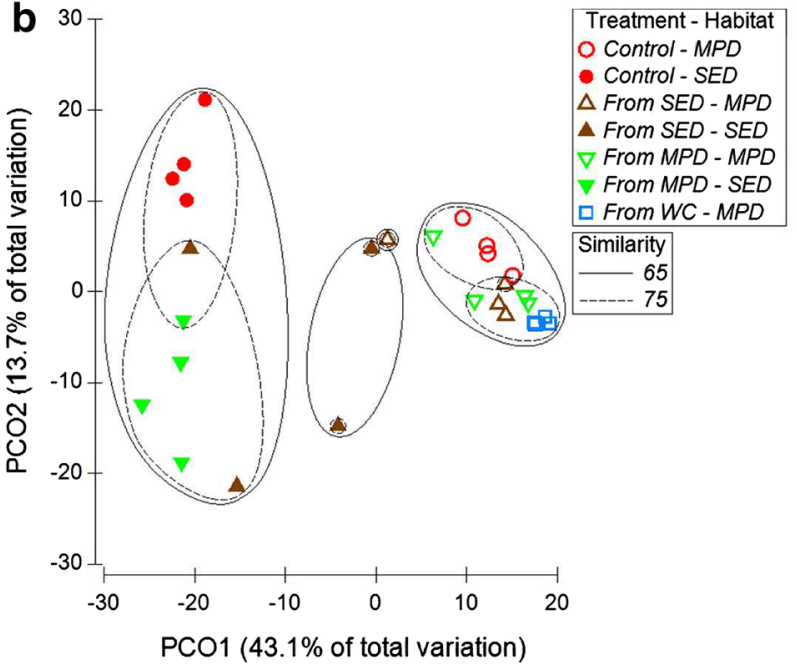

ent treatments are represented by symbols: circles control; triangles from SED; reverse triangles from MPD; squares from WC (water column). Full line represents $65 \%$ similarity and dashed line represents $75 \%$ similarity

'Control' compartments after $96 \mathrm{~h}$ of incubation (Pairwise: $t=2.92, P_{(\mathrm{MC})}=0.025$ for MPD and $t=2.68$, $P_{(\mathrm{MC})}=0.031$ for SED). In terms of species composition, pairwise comparisons revealed significant differences between the $T_{96}$ 'Control' habitats and the $T_{0}$ reference samples (Table 3). The dissimilarity between the $T_{0}$ and the $T_{96}$ 'Control' reached $53.5 \%$ for the MPD and $52.9 \%$ for the SED habitats (SIMPER). 


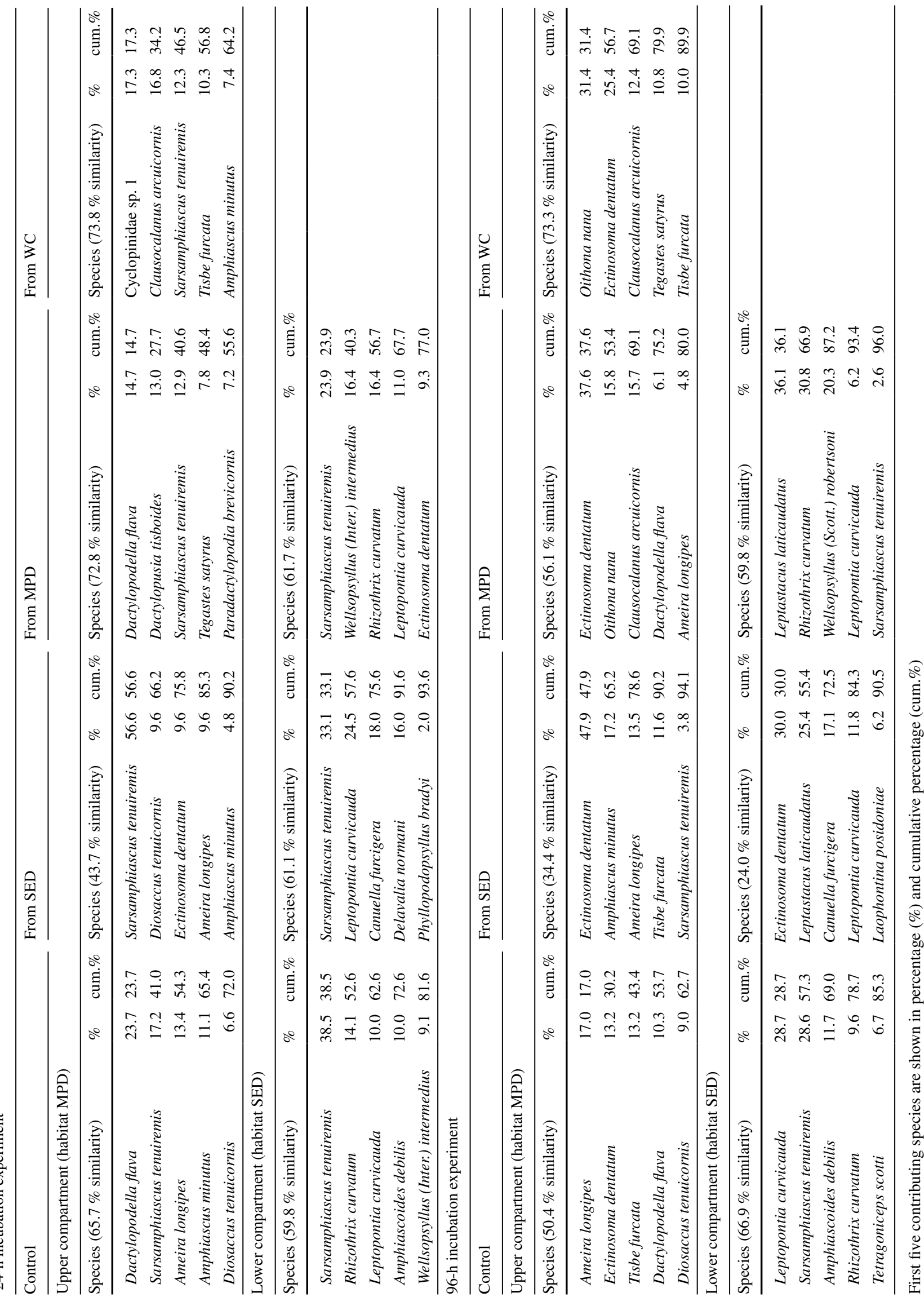


Table 5 Diversity metrics (average $\pm \mathrm{SD}$ ) based on $N=4$ per treatment per compartment for both incubation experiments
$S$ number of species observed, $d$ Margalev's species richness, $H^{\prime}$ Shannon species diversity, $E_{H}$ Heip's evenness index and $N_{1}$ abundance of the most dominant species. MPD macrophytodetritus, $S E D$ sediments and $W C$ water column 24-h incubation experiment

\begin{tabular}{lllll}
\hline$T_{0}$ & $T_{24}$ & $T_{24}$ & $T_{24}$ & $T_{24}$
\end{tabular}

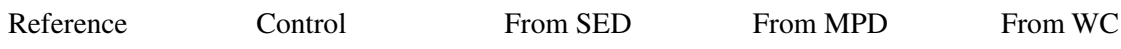

\begin{tabular}{lrrrrr}
\hline \multicolumn{5}{l}{ Upper compartment (habitat MPD) } \\
$S$ & $25.25 \pm 0.96$ & $23.75 \pm 0.96$ & $8 \pm 0.82$ & $23.67 \pm 2.08$ & $21 \pm 1.83$ \\
$d$ & $5.07 \pm 0.23$ & $4.32 \pm 0.18$ & $2.43 \pm 0.09$ & $4.37 \pm 0.33$ & $4.39 \pm 0.42$ \\
$H^{\prime}$ & $2.82 \pm 0.07$ & $2.87 \pm 0.06$ & $2.37 \pm 0.26$ & $2.9 \pm 0.06$ & $1.64 \pm 0.1$ \\
$E_{\mathrm{H}}$ & $0.25 \pm 0.01$ & $0.29 \pm 0.02$ & $0.58 \pm 0.15$ & $0.3 \pm 0.03$ & $0.1 \pm 0.01$ \\
$N_{1}$ & $16.78 \pm 1.13$ & $17.74 \pm 1.08$ & $10.94 \pm 3.06$ & $18.27 \pm 1.15$ & $5.18 \pm 0.49$
\end{tabular}

Lower compartment (habitat SED)

$\begin{array}{lrrrr}S & 14.33 \pm 0.58 & 12.64 \pm 5.1 & 10.25 \pm 0.5 & 14 \pm 1.73 \\ d & 3.6 \pm 0.29 & 3.03 \pm 0.92 & 2.31 \pm 0.19 & 3.12 \pm 0.35 \\ H^{\prime} & 2.24 \pm 0.13 & 2.14 \pm 0.46 & 1.9 \pm 0.06 & 2.76 \pm 0.07 \\ E_{\mathrm{H}} & 0.26 \pm 0.04 & 0.32 \pm 0.11 & 0.27 \pm 0.03 & 0.45 \pm 0.05 \\ N_{1} & 9.49 \pm 1.21 & 9.19 \pm 4.09 & 6.73 \pm 0.39 & 15.79 \pm 1.1\end{array}$

96-h incubation experiment

\begin{tabular}{lllll}
\hline$T_{0}$ & $T_{96}$ & $T_{96}$ & $T_{96}$ & $T_{96}$ \\
Reference & Control & From SED & From MPD & From WC
\end{tabular}

Upper compartment (habitat MPD)

\begin{tabular}{lrrrrr}
$S$ & $25.25 \pm 0.96$ & $17 \pm 4.4$ & $8 \pm 2.83$ & $17 \pm 3.92$ & $19 \pm 2.71$ \\
$d$ & $5.07 \pm 0.23$ & $4.38 \pm 0.65$ & $2.53 \pm 0.81$ & $3.78 \pm 0.55$ & $3.59 \pm 0.45$ \\
$H^{\prime}$ & $2.82 \pm 0.07$ & $2.62 \pm 0.24$ & $1.84 \pm 0.34$ & $2.37 \pm 0.31$ & $2.16 \pm 0.21$ \\
$E_{\mathrm{H}}$ & $0.25 \pm 0.01$ & $0.32 \pm 0.02$ & $0.37 \pm 0.09$ & $0.26 \pm 0.05$ & $0.18 \pm 0.02$ \\
$N_{1}$ & $16.78 \pm 1.13$ & $13.99 \pm 3.34$ & $6.57 \pm 1.99$ & $11.12 \pm 3.85$ & $8.82 \pm 1.83$ \\
\multicolumn{5}{l}{ Lower compartment (habitat SED) } \\
$S$ & $14.33 \pm 0.58$ & $10.75 \pm 0.96$ & $6 \pm 2$ & $9.25 \pm 2.22$ & \\
$d$ & $3.6 \pm 0.29$ & $2.74 \pm 0.25$ & $1.92 \pm 0.25$ & $2.08 \pm 0.6$ & \\
$H^{\prime}$ & $2.24 \pm 0.13$ & $2.06 \pm 0.13$ & $1.6 \pm 0.16$ & $1.65 \pm 0.23$ & \\
$E_{\mathrm{H}}$ & $0.26 \pm 0.04$ & $0.3 \pm 0.06$ & $0.39 \pm 0.08$ & $0.24 \pm 0.04$ & \\
$N_{1}$ & $9.49 \pm 1.21$ & $7.91 \pm 0.98$ & $4.87 \pm 0.69$ & $5.33 \pm 1.22$ & \\
\hline \multicolumn{5}{r}{} \\
\end{tabular}

Colonization of defaunated macrophytodetritus from the sediments (treatment 'From SED') took place. After $96 \mathrm{~h}$, $7.2 \pm 7.0$ indiv. $\mathrm{g}^{-1} \mathrm{DW}$ were found; however, a large variability amongst the replicates was present (Fig. 2b). The species composition present in the newly colonized MPD habitat showed no significant difference (Table 3) compared to the 'Control'. Nonetheless, a dissimilarity of $68.6 \%$ (SIMPER) was present. The newly colonized habitat was dominated by Ectinosoma dentatum (Ectinosomatidae family) (Table 4). In terms of species number, diversity and richness, lower values were noted compared to the 'Control'. However, a larger evenness was found (Table 5). In the natural sediment, the colonisers' source compartment, two species dominated after $96 \mathrm{~h}$ : the E. dentatum and Leptastacus laticaudatus (Table 4). Again a low species number, diversity and richness were combined with a high evenness (Table 5).

Colonization of the defaunated sediments by copepods from the natural macrophytodetritus was effective within
$96 \mathrm{~h}$. The copepod densities of the colonized habitat in the treatment 'From MPD' increase to $7.4 \pm 0.8$ indiv. $10 \mathrm{~cm}^{-2}$ with species diversity metrics, showing no significant differences with the 'Control' (Fig. 2b). However, the species composition was significantly different (Table 3). Two species were most abundant in the SED compartment: L. laticaudatus and Rhizotrix curvatum (Table 4). The colonisers' source MPD compartment showed, in spite of a decrease in density towards $5.8 \pm 2.3$ indiv. $\mathrm{g}^{-1} \mathrm{DW}$, a similar diversity and species composition as the 'Control' MPD habitat. Worth noticing are the two non-harpacticoid species Oithona nana and Clausocalanus arcuicornis present in the top five contributors of the similarity (Table 4).

The colonization of defaunated macrophytodetritus from the water column, treatment 'From WC', displayed a strong increase in copepods density from zero to $35.4 \pm 9.4$ indiv. $\mathrm{g}^{-1} \mathrm{DW}$, which is more than double of the densities after $24 \mathrm{~h}$ (Fig. 2b). After 96-h incubation, 
similar species numbers and richness were found, however, with a higher evenness and diversity compared to the 24-h incubation. The species composition changed and another Cyclopoid copepod, $O$. nana, became dominant closely followed by E. dentatum (Table 4).

\section{Discussion}

\section{Macrophytodetritus colonization}

Our results showed colonization of all defaunated habitats by species originating from adjacent habitats. These adjacent habitats were composed of very specific communities and species compositions defining each adjacent habitat. The densities and diversities encountered during the experiment were congruent with previous studies in the area (Dauby 1980; Heip et al. 1983; Mascart et al. 2013, 2015).

Colonization occurred from adjacent habitats within the first $24 \mathrm{~h}$, which is corresponding to recovery times found after a physical disturbance (Sun and Fleeger 1994; Fleeger et al. 1995). Meiobenthic copepods exert colonization and active habitat selection via two pathways: infaunal dispersion through the interstitial spaces and dispersion through suspension in the water column. The majority of adjacent habitat-specific typifying species were present in the macrophytodetritus, therefore showing traits of dispersion and making the macrophytodetritus a diverse copepod hub. Moreover, some species seemed to be exclusively associated with macrophytodetritus. It could thus be hypothesized that species have more than one generation in these provisional accumulations, highlighting the ecological role of macrophytodetritus to the overall seagrass system.

When defaunated macrophytodetritus covered natural bare sediments, an almost immediate interstitial colonization through the boundary layer from the sediment community occurred. Nonetheless, the formed assemblage did not fully resemble the possible macrophytodetritus assemblage as found in the control. Similar patterns were found in the colonization from the water column towards defaunated macrophytodetritus. On the contrary, during colonization from the natural macrophytodetritus towards defaunated sediments, the formed assemblage in the sediment resembled the control as shown in the PCO (Fig. 3a). It can therefore be concluded, firstly, that species defining the sedimentary assemblage were present in the natural macrophytodetritus and crossed the boundary layer downwards. Secondly, that infaunal colonization through the interstitial spaces played a major role, in the occurrence of two contiguous habitats. In case two habitats were not contiguous, recruitment occurred through dispersal through the water column. Thirdly, assemblages in macrophytodetritus were a mixture of the surrounding habitat assemblages.
Subsequently, not only habitat-specific species actively migrate as suggested by Hicks (1986). This study concluded that ecologically different copepods originating from diverse habitats were conspicuous dispersers and actively migrated towards defaunated habitats using their species-specific preferred dispersal pathways.

\section{Dispersion and colonization drivers}

In our study, hydrodynamic flow effects were excluded, due to the experimental set-up shielding the macrophytodetritus. Nonetheless, in natural environments, the recruitment or settlement of water-bound benthic organisms is defined by landscape attributes and hydrodynamic processes (Armonies 1994; Commito and Tita 2002). Palmer (1984) states that meiofauna inhabiting unvegetated habitats avoid the benthic boundary layer during high flow disturbances and frequent the sediment surface during reduced flow. Since above-ground structures locally reduce the hydrodynamic disturbance and diminish the predation risk associated with freely swimming in the water column (Coull and Wells 1983; Palmer and Gust 1985), macrophytodetritus stabilizes the hydrodynamic flow and serves as refuge from predators. However, macrophytodetritus is only deposited on the seafloor and is not rooted. Therefore, during low hydrodynamic flow, it indeed provides shelter from flow and predation, to the contrary during high hydrodynamic flow or storms, the macrophytodetritus and the upper layer of sediment passively (re)suspends in the water column together with their associated organisms (Thistle et al. 1995). Subsequently, the suspended material resettles randomly on different sand patches, driven by hydrodynamic flows. Hence, rafting or drifting on macrophytodetritus triggered by storms should be considered as a dispersal method at larger spatial scales (Bonsdorff 1992; Norkko and Bonsdorff 1996; Ólafsson et al. 2001).

High disturbances have an adverse effect on densities; however, intermediate disturbances could have an opposing effect, corresponding to the intermediate disturbance hypothesis (Connell 1978; Cadotte 2007). It states that high diversity is a consequence of continually changing conditions. As a result, disturbance is put forward as the explanation for coexistence of species and often high disturbance resets the local succession pathway (Connell 1978). Under sheltered conditions, macrophytodetritus interstitial water slowly changes from water-column-like chemical conditions to oxygen-poor conditions, due to bacterial respiration and reduced compound advection from sediment (Mascart et al. 2015). Community structure significantly changed between the $\mathrm{T}_{0}$ reference samples and the $T_{96}$ 'Control' samples, without showing a drop in densities. For macrofauna, Gallmetzer et al. (2005) and Remy (unpublished data) found a dominance shift towards low-oxygen tolerant 
species, reducing the overall diversity in the macrophytodetritus accumulation under stable conditions. Therefore, the experimental units are not expected to have a negative impact on a short-term interval. However, on the long term, a plausible oxygen drop could occur and perturb the initial community structure. Hence, the $T_{96}$ incubations displayed the variability of the community structure and its sensibility to a potential drop in oxygen levels.

The habitat selectivity of the copepod resettlement (following a passive erosion or active emergence) depends on the chemical and microbiological signals perceived at small distances (Hicks 1977; Fleeger et al. 1995). Decho and Fleeger (1988) stated that copepods exhibit a preference for food-enriched microhabitats over their initial habitat food availability. Macrophytodetritus are abundantly colonized by microepiphytes (diatoms, bacteria, fungi, protists) (Lepoint et al., 2006) degrading detrital material or using remineralized nutrients. Macrophytodetritus could support a higher microbial biomass and production than living material, since it may have reduced levels of polyphenolic compounds, which in living leaves deter both bacterial colonization and herbivory (Dethier et al. 2014). Degrading seagrass material could be also a minor source of food for few species (Mascart et al. 2013).

In conclusion, our study is congruent with Bostrom and Bonsdorff (2000), whom stated that structurally complex plant assemblages (like macrophytodetritus) may trap or attract organisms more efficiently than structurally simple leaf canopies or bare sediments. Structural complexity and dispersion drivers, such as hydrodynamics (i.e. disturbances), habitat complexity (i.e. shelter effect) and food availability, control the colonization ability of a copepod population.

Species-specific behaviour and ecological types

This study rigorously tried to quantify and qualify the species-specific active colonization of copepods from adjacent habitats towards defaunated habitats. Our results showed that the dominant Sarsamphiascus tenuiremis (Miraciidae family) rapidly colonized macrophytodetritus, using two different dispersal pathways, one via the water column and another via the sediment and macrophytodetritus interstitial spaces. In order to be able to use the former pathway, one needs to actively emerge from the substrate's surface (e.g. seagrass leafs or sediment) and possess well-developed swimming abilities to than disperse (Thistle and Sedlacek 2004). Other genera, for instance, Ambunguipes, Ameira, Amphiascoides, Amphiascus, Dactylopodella, Diosaccus and Ectinosoma seemed to have a similar behaviour in our study. On the other hand, the genera Arenosetella, Canuella, Leptastacus, Leptopontia, Rhizotrix, Tetragoniceps and Wellsopsyllus did not seem to behave in an emergent way and preferably stayed in the sediment where they can interstitially migrate between bordering substrata. This is congruent with the study of Hockin and Ollason (1981) whom differentiated two sets of species, one with superior and one with inferior dispersal abilities. Indeed, several of the above-mentioned species were exclusively present in the sediments, showing no tendency to migrate and therefore can be tagged as species with inferior dispersal abilities. Regarding the species with superior dispersion abilities Kurdziel and Bell (1992) defined seven true phytal-dwelling families on Thalassia testudinum in accordance with previous research by Hicks and Coull (1983). Although studying a temperate water seagrass meadow, our results were analogous as those families were all present in the $P$. oceanica canopy and both studies share the same dominant genus, Harpacticus. The P. oceanica phytal-dwelling species contributed for $50 \%$ to the species composition of naturally occurring macrophytodetritus. The prominent role of seagrass species can partially be explained by the origin of macrophytodetritus. Phytal copepods have prehensile legs, allowing them to cling to a substrate (Hicks and Coull 1983); therefore, it can be assumed that some species are passively transported attached on the falling dead leaves.

Besides, Kurdziel and Bell (1992) found free-swimming Cyclopoids in the T. testudinum canopies. Our study found similar copepods in the macrophytodetritus together with calanoids. Hence, free-swimming species can play an important role and represent up to $30-40 \%$ in the densities of the macrophytodetritus assemblage. It is known that these copepods migrate vertically to the bottom for shelter, reproduction and feeding purposes (Teasdale et al. 2004). Seen their high abundance in the 'From WC' treatment, especially with time, it should not be excluded that macrophytodetritus has an important ecological role for freeswimming copepods from coastal area.

Consequently, macrophytodetritus species assemblages are composed of passive leaf clingers, active infaunal dispersers, active suspended dispersers and free-swimming copepods. All those different dispersion modes of adult copepods seem to confirm their importance to population dispersion. Chandler and Fleeger (1983) concluded in their colonization experiment, which suspended water column dispersion is more important than interstitial infaunal transport. However, the latter should not be neglected. Every species with good swimming capacities and capability of emergence (e.g. Armonies, 1994; Kurdziel and Bell 1992; Thistle and Sedlacek, 2004) colonized or showed a predisposition to migrate to new or provisional habitats. Therefore, it would be preferable to refine the provisional classification of Noodt (1971) with a more ecological and mobilitybased grouping. Subsequently, in Table 1, the active colonization capabilities of some species were incorporated. 
Hicks (1986) predicted the epibenthic capabilities of many phytal species, however, he agreed upon Palmer and Gust (1985) ideas of passive erosion being the only pathway to suspend benthic copepods from unvegetated habitats. The presented results prove that active emergence and migration occur from sedimentary habitats, water column habitats and phytal habitats. Therefore, it would be desirable to correct the idea that exclusively phytal components actively contribute members to the water column, as the results proved that not only phytal-dwelling copepods possess well-developed swimming abilities.

The formation of the communities during low disturbance conditions seems to distinguish two stages. The initial stage is characterized by colonizer species and subsequently after a few days competitor species arrive on site, impacting the subsequent settlement (Sun and Fleeger 1994; Jacobi and Langevin 1996). Colonization experiments over time (e.g. Chertoprud et al. 2005; De Troch et al. 2005) generally observed a stage shift after more than 4 days, the duration of the second incubation experiment. However, an initiation of a stage shift seemed visible in our 96-h experiment. Ectinosoma dentatum became the most dominant species in the newly colonized macrophytodetritus after $96 \mathrm{~h}$. Hence, a colonization-competition trade-off seemed to be present between S. tenuiremis and E. dentatum. However, in the reference samples, $S$. tenuiremis is more abundant than $E$. dentatum. During a seasonal characterization study in the same bay (Mascart et al. 2015), effects of oxygen gradients were measured for Diosaccus tenuicornis, a species from the same Miraciidae family as $S$. tenuiremis. To the contrary, E. dentatum seemed not to be impacted by the oxygen level present in the macrophytodetritus. De Troch et al. (2005) confirmed the definition of Hicks and Coull (1983) of Ectinosomatidae as 'jacks of all habitats', so-called r-strategist. The experiment revealed thus a sensibility of $S$. tenuiremis towards a potential drop in oxygen gradient induced by the experimental setup after $96 \mathrm{~h}$. This could explain the difference between the control and the reference samples after $96 \mathrm{~h}$. Therefore, it can be concluded that the experiment duration was not long enough to see a stage shift, but an effect of drop in oxygen was visible. Nonetheless, it confirms the important role and flexibility of $E$. dentatum within the macrophytodetritus accumulations and the main colonizer role for $S$. tenuiremis. Both E. dentatum and $S$. tenuiremis used the infaunal and suspension pathway for active colonization.

\section{Conclusions}

In conclusion, free-living harpacticoid copepods actively colonize adjacent defaunated macrophytodetritus and sediment within 24 h. Eco-morphologically different copepods originating from diverse habitats (sediment, phytal and pelagic) were conspicuous dispersers and actively migrated towards defaunated habitats using their species-specific preferred dispersal pathways. Two pathways occur for active colonization: infaunal migration through the boundary layer and migration suspended via the water column. Eco-morphological characteristics can be used to predict the potential preferred habitat of copepods. However, copepods are not obligate residents of their preferred habitat since phytal and sedimentary components actively contribute to the water column. Therefore, copepods are opportunistic and are capable of assembling dynamic communities in provisional habitats. Macrophytodetritus offers a structurally complex substitute habitat proposing shelter and food supply, therefore serving as a hub for all copepods of the surrounding habitats. Due to the high diversity of associated fauna, the macrophytodetritus assemblage has a certain resilience which can cope and restore swiftly from disturbances. Swimming abilities, structural complexity and dispersion drivers, such as hydrodynamics, habitat complexity and food availability, control the colonization ability of a copepod population.

Acknowledgments The authors kindly thank the Stareso field station staff for their assistance in the experimental setup and Renzo Biondo for his help throughout the design. The authors thank the three anonymous referees for their constructive remarks that contributed to the improvement of the manuscript. The first author acknowledges a F.R.I.A. Ph.D. Grant (Belgian National Fund for Research Training in industry and in agriculture). This study was conducted within the frame of FRS-FNRS research Project FRFC 2.4511.09 (University of Liège) with additional support provided by the Ghent University (BOF-GOA 01GZ0705 and 01GA1911W). G. Lepoint is a Research Associate of the FRS-FNRS. M. De Troch is a postdoctoral fellow financed by the Special Research Fund of the Ghent University (BOF-GOA 01GA1911 W). This is the MARE paper number 288.

\section{References}

Anderson MJ, Gorley RN, Clarke KR (2008) PERMANOVA+ for PRIMER: guide to software and statistical methods. PRIMER-E, Plymouth

Armonies W (1994) Drifting meio- and macrobenthic invertebrates on tidal flats in Königshafen: a review. Helgolander Meeresunters 48:299-320. doi:10.1007/BF02367043

Bell SS, Walters K, Hall MO (1987) Habitat utilization by harpacticoid copepods-a morphometric approach. Mar Ecol Prog Ser 35:59-64. doi:10.3354/meps035059

Bodin P, Leguellec C (1992) Meiobenthos of the bay of Saint-Brieuc (North Brittany, France). 2. Harpacticoid copepod diversity and species assemblages. Oceanol Acta 15:673-686

Bongers T (1990) The maturity index: an ecological measure of environmental disturbance based on nematode species composition. Oecologia 83:14-19. doi:10.1007/bf00324627

Bonsdorff E (1992) Drifting algae and zoobenthos-effects on settling and community structure. Neth J Sea Res 30:57-62. doi:10.1016/0077-7579(92)90045-g 
Bostrom C, Bonsdorff E (2000) Zoobenthic community establishment and habitat complexity - the importance of seagrass shoot-density, morphology and physical disturbance for faunal recruitment. Mar Ecol Prog Ser 205:123-138

Bostrom C, Tornroos A, Bonsdorff E (2010) Invertebrate dispersal and habitat heterogeneity: expression of biological traits in a seagrass landscape. J Exp Mar Biol Ecol 390:106-117. doi:10.1016/j. jembe.2010.05.008

Boxshall GA, Hasley SH (2004) An introduction to copepods diversity. The Ray Society, London

Cadotte MW (2007) Competition-colonization trade-offs and disturbance effects at multiple scales. Ecology 88:823-829. doi:10.1890/06-1117

Callens M, Gheerardyn H, Ndaro SGM, De Troch M, Vanreusel A (2012) Harpacticoid copepod colonization of coral fragments in a tropical reef lagoon (Zanzibar, Tanzania). J Mar Biol Assoc U K 92:1535-1545. doi:10.1017/s0025315411001597

Chandler GT, Fleeger JW (1983) Meiofaunal colonization of azoic estuarine sediment in Louisiana: mechanisms of dispersal. J Exp Mar Biol Ecol 69:175-188. doi:10.1016/0022-0981(83)90066-7

Chertoprud ES, Azovsky AI, Sapozhnikov FV (2005) Colonization of azoic sediments of different grain-size composition by littoral Harpacticoida : Copepoda. Oceanology 45:698-706

Clarke KR, Gorley RN (2006) PRIMER v6. In: PRIMER-E (ed) User Manual/Tutorial, Plymouth

Commito JA, Tita G (2002) Differential dispersal rates in an intertidal meiofauna assemblage. J Exp Mar Biol Ecol 268:237-256. doi:10.1016/S0022-0981(01)00386-0

Connell JH (1978) Diversity in tropical rain forests and coral reefs. Science 199:1302-1310. doi:10.1126/science.199.4335.1302

Coull BC, Wells JBJ (1983) Refuges from fish predation-experiments with phytal meiofauna from the New-Zealand rocky intertidals. Ecology 64:1599-1609. doi:10.2307/1937513

Danovaro R, Fraschetti S (2002) Meiofaunal vertical zonation on hard-bottoms: comparison with soft-bottom meiofauna. Mar Ecol Prog Ser 230:159-169. doi:10.3354/meps230159

Dauby P (1980) Cycle annuel du zooplancton de surface de la baie de Calvi (Corse). Biomasse totale et plancton copépodien. Acta Oecol 3:403-407

De Troch M, Gurdebeke S, Fiers F, Vincx M (2001) Zonation and structuring factors of meiofauna communities in a tropical seagrass bed (Gazi Bay, Kenya). J Sea Res 45:45-61. doi:10.1016/ S1385-1101(00)00055-1

De Troch M, Vandepitte L, Raes M, Suarez-Morales E, Vincx M (2005) A field colonization experiment with meiofauna and seagrass mimics: effect of time, distance and leaf surface area. Mar Biol 148:73-86. doi:10.1007/s00227-005-0062-x

Decho AW, Fleeger JW (1988) Microscale dispersion of meiobenthic copepods in response to food-resource patchiness. J Exp Mar Biol Ecol 118:229-243. doi:10.1016/0022-0981(88)90075-5

Dethier MN, Brown AS, Burgess S, Eisenlord ME, Galloway AWE, Kimber J, Lowe AT, O'Neil CM, Raymond WW, Sosik EA, Duggins DO (2014) Degrading detritus: changes in food quality of aging kelp tissue varies with species. J Exp Mar Biol Ecol 460:72-79. doi:10.1016/j.jembe.2014.06.010

Faust MA, Gulledge RA (1996) Associations of microalgae and meiofauna in floating detritus at a mangrove island, Twin Cays, Belize. J Exp Mar Biol Ecol 197:159-175

Fleeger JW, Chandler GT, Fitzhugh GR, Phillips FE (1984) Effects of tidal currents on meiofauna densities in vegetated salt marsh sediments. Mar Ecol Prog Ser 19:49-53. doi:10.3354/ meps019049

Fleeger JW, Yund PO, Sun B (1995) Active and passive processes associated with initial settlement and post-settlement dispersal of suspended meiobenthic copepod. J Mar Res 53:609-645. doi: $10.1357 / 0022240953213070$
Frisch D, Green AJ (2007) Copepods come in first: rapid colonization of new temporary ponds. Fundam Appl Limnol 168:289-297. doi:10.1127/1863-9135/2007/0168-0289

Gallmetzer I, Pflugfelder B, Zekely J, Ott JA (2005) Macrofauna diversity in Posidonia oceanica detritus: distribution and diversity of mobile macrofauna in shallow sublittoral accumulations of Posidonia oceanica detritus. Mar Biol 147:517-523. doi:10.1007/ s00227-005-1594-9

Gheerardyn H, de Troch M, Vincx M, Vanreusel A (2009) Harpacticoida (Crustacea: Copepoda) associated with cold-water coral substrates in the Porcupine Seabight (NE Atlantic): species composition, diversity and reflections on the origin of the fauna. Sci Mar 73:747-760. doi:10.3989/scimar.2009.73n4747

Giere O (2009) Meiobenthology - the microscopic motile fauna of aquatic sediments. Springer, Berlin

Guidi-Guilvard LD, Thistle D, Khripounoff A, Gasparini S (2009) Dynamics of benthic copepods and other meiofauna in the benthic boundary layer of the deep NW Mediterranean Sea. Mar Ecol Prog Ser 396:181-195. doi:10.3354/meps08408

Hagerman GM, Rieger RM (1981) Dispersal of benthic meiofauna by wave and current action in bogue sound, North Carolina, USA. Mar Ecol 2:245-270. doi:10.1111/j.1439-0485.1981.tb00099.x

Hamner WM, Carleton JH (1979) Copepod swarms: attributes and role in coral reef ecosystems. Limnol Oceanogr 24:1-14

Heip C, Willems K, Van hauwermeiren G (1983) Een systematischecologische studie van Copepoda Harpacticoida levend op bruinwieren van het genus Cystoseira in de Baai van Calvi (Corsica). LUC, Diepenbeek

Hicks GRF (1977) Observations on substrate preference of marine phytal harpacticoids (copepoda). Hydrobiologia 56:7-9

Hicks GRF (1980) Structure of phytal harpacticoid copepod assemblages and the influence of habitat complexity and turbidity. $\mathbf{J}$ Exp Mar Biol Ecol 44:157-192

Hicks GRF (1986) Distribution and behaviour of meiofaunal copepods inside and outside seagrass beds. Mar Ecol Prog Ser 31:159170. doi: $10.3354 /$ meps031159

Hicks GRF, Coull BC (1983) The ecology of marine meiobenthic harpacticoid copepods. Oceanogr Mar Biol 21:67-175

Higgins R, Thiel H (1988) Introduction to the study of meiofauna. Smithsonian Institution Press, London

Hockin DC, Ollason JG (1981) The colonization of artificially isolated volumes of intertidal estuarine sand by harpacticoid copepods. J Exp Mar Biol Ecol 53:9-29. doi:10.1016/0022-0981(81)90081-2

Hulings NC, Gray JS (1971) A manual for the study of meiofauna. Smithson Contrib Zool 78:1-83

Huys R, Boxshall GA (1991) Copepod evolution. London Ray Society, London

Jacobi CM, Langevin R (1996) Habitat geometry of benthic substrata: effects on arrival and settlement of mobile epifauna. J Exp Mar Biol Ecol 206:39-54. doi:10.1016/s0022-0981(96)02605-6

Kiorboe T (2000) Colonization of marine snow aggregates by invertebrate zooplankton: abundance, scaling, and possible role. Limnol Oceanogr 45:479-484

Koski M, Kiorboe T, Takahashi K (2005) Benthic life in the pelagic: aggregate encounter and degradation rates by pelagic harpacticoid copepods. Limnol Oceanogr 50:1254-1263

Kotwicki L, De Troch M, Urban-Malinga B, Gheskiere T, Weslawski JM (2005) Horizontal and vertical distribution of meiofauna on sandy beaches of the North Sea (The Netherlands, Belgium, France). Helgol Mar Res 59:255-264. doi:10.1007/ s10152-005-0001-8

Kurdziel JP, Bell SS (1992) Emergence and dispersal of phytaldwelling meiobenthic copepods. J Exp Mar Biol Ecol 163:43-64. doi:10.1016/0022-0981(92)90146-2

Lang K (1948) Monographie der Harpacticiden. Hâkan Ohlssons boktryckeri, Lund 
Lepoint G, Cox AS, Dauby P, Poulicek M, Gobert S (2006) Food sources of two detritivore amphipods associated with the seagrass Posidonia oceanica leaf litter. Mar Biol Res 2:355-365. doi:10.1080/17451000600962797

Magurran AE (2004) Measuring biological diversity. Blackwell Publishing, Oxford

Magurran AE, McGill BJ (2011) Biological diversity: frontiers in measurement and assessment. Oxford University Press, New York

Mascart T, Lepoint G, De Troch M (2013) Meiofauna and harpacticoid copepods in different habitats of a Mediterranean seagrass meadow. J Mar Biol Assoc UK 93:1557-1566. doi:10.1017/ s0025315413000222

Mascart T, Lepoint G, Deschoemaeker S, Binard M, Remy F, De Troch M (2015) Seasonal variability of meiofauna, especially harpacticoid copepods, in Posidonia oceanica macrophytodetritus accumulations. J Sea Res 95:149-160. doi:10.1016/j.seares.2014.07.009

Mateo MA, Romero J (1997) Detritus dynamics in the seagrass Posidonia oceanica: elements for an ecosystem carbon and nutrient budget. Mar Ecol Prog Ser 151:43-53. doi:10.3354/meps 151043

McIntyre AD (1969) Ecology of marine meiobenthos. Biol Rev 44:245-288. doi:10.1111/j.1469-185X.1969.tb00828.x

Noodt W (1971) Ecology of the Copepoda. Smithsonian Institution Press, Washington

Norkko A, Bonsdorff E (1996) Rapid zoobenthic community responses to accumulations of drifting algae. Mar Ecol Prog Ser 131:143-157. doi:10.3354/meps131143

Ólafsson E, Ingólfsson A, Steinarsdóttir MB (2001) Harpacticoid copepod communities of floating seaweed: controlling factors and implications for dispersal. Hydrobiologia 453-454:189-200

Palmer MA (1984) Invertebrate drift: behavioral experiments with intertidal meiobenthos. Mar Behav Physiol 10:235-253

Palmer MA (1988) Dispersal of marine meiofauna a review and conceptual model explaining passive transport and active emergence with implications for recruitment. Mar Ecol Prog Ser 48:81-91. doi:10.3354/meps048081

Palmer MA, Gust G (1985) Dispersal of meiofauna in a turbulent tidal creek. J Mar Res 43:179-210

Quinn GGP, Keough MJ (2002) Experimental design and data analysis for biologists. Cambridge University Press, Cambridge

Remane A (1952) Die Besiedlung des Sandbodens im Meere und die Bedeutung der Lebensformtypen für die Ökologie. Verhandlungen der Deutschen Zoologischen Gesellschaft 1951:327-359

Romero J, Pergent G, Pergentmartini C, Mateo MA, Regnier C (1992) The detritic compartment in a Posidonia oceanica meadow-litter features, decomposition rates and mineral stocks. Mar Ecol 13:69-83
Sanmarti N, Menendez M (2007) Litter decomposition of Scirpus maritimus L. in a Mediterranean coastal marsh: importance of the meiofauna during the initial phases of detached leaves decomposition. Int Rev Hydrobiol 92:211-226. doi:10.1002/ iroh.200510955

Scheef LP, Marcus NH (2010) Occurrence and significance of copepod resting egg accumulation in seagrass sediments. Mar Ecol Prog Ser 407:125-134. doi:10.3354/meps08573

Sedlacek L, Thistle D (2006) Emergence on the continental shelf: differences among species and between microhabitats. Mar Ecol Prog Ser 311:29-36. doi:10.3354/meps311029

Sun B, Fleeger JW (1994) Field experiments on the colonization of meiofauna into sediment depressions. Mar Ecol Prog Ser 110:167-175. doi:10.3354/meps110167

Teasdale M, Vopel K, Thistle D (2004) The timing of benthic copepod emergence. Limnol Oceanogr 49:884-889

Thielemans LKH, Heip C (1984) The response of a harpacticoid copepod community to sediment disturbance in a semi-enclosed lagoon. Hydrobiologia 118:127-133. doi:10.1007/bf00031795

Thistle D (1978) Harpacticoid dispersion patterns implications for deep-sea diversity maintenance. J Mar Res 36:377-397

Thistle D, Sedlacek L (2004) Emergent and non-emergent species of harpacticoid copepods can be recognized morphologically. Mar Ecol Prog Ser 266:195-200. doi:10.3354/meps266195

Thistle D, Weatherly GL, Wonnacott A, Ertman SC (1995) Suspension by winter storms has an energetic cost for adult male benthic harpacticoid copepods at a shelf site. Mar Ecol Prog Ser 125:7786. doi: $10.3354 /$ meps 125077

Thomsen MS, Muth MF, McGlathery KJ (2011) Tube-forming polychaetes enhance invertebrate diversity and abundance in sandy sediments of Mozambique, Africa. Afr J Mar Sci 33:327-332. doi $: 10.2989 / 1814232 x .2011 .600433$

Torres-Pratts H, Schizas NV (2007) Meiofaunal colonization of decaying leaves of the red mangrove Rhizophora mangle, in southwestern Puerto Rico. Caribb J Sci 43:127-137

Vetter EW (1995) Detritus-based patches of high secondary production in the nearshore benthos. Mar Ecol Prog Ser 120:251-262. doi:10.3354/meps 120251

Walters K (1991) Influences of abundance, behavior, species composition, and ontogenetic stage on active emergence of meiobenthic copepods in subtropical habitats. Mar Biol 108:207-215. doi:10.1007/BF01344335

Walters K, Bell SS (1986) Diel patterns of active vertical migration in seagrass meiofauna. Mar Ecol Prog Ser 34:95-103. doi:10.3354/ meps034095 